\title{
Validity and reliability of standing balance control measurement on the left unilateral spatial neglect model
}

\author{
ABDUL C. MEIDIAN ${ }^{1,2}$ | SONG YIGE ${ }^{1}$ | SYAHMIRZA I. LESMANA² | KAZU AMIMOTO1 \\ 1 Department of Physical Therapy, Graduate School of Human Health Sciences, Tokyo Metropolitan University, Tokyo, Japan. \\ ${ }^{2}$ Faculty of Physiotherapy, Esa Unggul University, Jakarta, Indonesia. \\ Correspondence to: Abdul Chalik Meidian. Department of Physical Therapy, Graduate School of Human Health Sciences, Tokyo Metropolitan University, Tokyo, 116-8551, \\ Japan. \\ email: achmeidian@gmail.com; meidian-abdulchalik@ed.tmu.ac.jp \\ https://doi.org/10.20338/bjmb.v15i3.247
}

\author{
HIGHLIGHTS \\ - We develop the HMD as a left unilateral \\ spatial neglect model in healthy subjects. \\ - Most CoP measurements have excellent \\ concurrent validity in regression analysis. \\ - A good agreement between the non-USN and \\ USN models showed in Bland-Altman plots. \\ - COP variability appeared to more reliable in \\ the frontal plane than sagittal plane. \\ - USN models use appeared to modulate \\ changes in postural adaptation and \\ adjustment.

$\begin{array}{ll}\text { ABBREVIATIONS } \\ \text { AP } & \text { Anterior-posterior } \\ \text { COP } & \text { Center of pressure } \\ \text { DSB } & \text { Dynamic standing balance } \\ \text { HMD } & \text { Head-mounted display } \\ \text { ICC } & \text { Intraclass correlation coefficient } \\ \text { L } & \text { Left } \\ \text { ML } & \text { Medial-lateral } \\ \text { MX } & \text { CoP alteration average on the X- } \\ & \text { axis } \\ \text { MY } & \text { CoP alteration average on the Y- } \\ & \text { axis } \\ \text { R } & \text { Right } \\ \text { SSB } & \text { Static standing balance } \\ \text { VR } & \text { Virtual reality } \\ \text { USN } & \text { Unilateral spatial neglect }\end{array}$

BACKGROUND: The present study developed a head-mounted display with the visual direction of a web camera modified to the right as a left unilateral spatial neglect (USN) model with respect to postural balance control. AIM: We aimed to estimate the validity and reliability of center of pressure (CoP) measurements in static standing balance (SSB) and dynamic standing balance (DSB) of healthy participants were using the USN model and to examine whether this model's use influenced postural balance control.

METHOD: A portable CoP force plate was used to quantify postural balance control in 64 healthy participants as the model. The CoP displacement of the non-USN and USN models in the medial-lateral (ML) and anteriorposterior (AP) planes, CoP length, and bilateral load ratio in SSB and DSB to the right (R) and left (L) were evaluated.

RESULTS: Regression analysis indicated that most COP measurements have excellent concurrent validity. Bland-Altman plots showed good agreement between the non-USN and USN models in the CoP measurements. Test-retest reliability estimation between two times measurements varied in the frontal and sagittal planes. A Comparison of the results demonstrated that the CoP-AP and CoP length changed $(-1.40 \%$ and $7.67 \%$, respectively) significantly in SSB $(P<0.05)$. Moreover, the CoP-AP changed very significantly in DSB-R and DSB-L $(-1.50 \%$ and $1.86 \%$, respectively) in opposite directions $(P<0.01)$ when the subjects performed as the model.

CONCLUSION: COP measurements are valid and reliable to quantify standing balance control in both non-USN and USN models that appear to modulate changes in postural adaptation and adjustment.

KEYWORDS: Validity | Reliability | Standing balance control | Center of pressure | Unilateral spatial neglect | Healthy participants

\section{INTRODUCTION}

Unilateral spatial neglect (USN) is a common behavioral disorder in right brain damage patients with sensory inattention to the contralateral side. ${ }^{1}$ Visual attention of the patient leads to only focus on the non-paretic side. 2,3 The USN condition causes a reduction of general responsivity and postural control adaptability so that the physical and behavioral consequences such as visual inattention, sensory-motor impairments, and postural imbalance impacting functional disability and rehabilitation outcomes. ${ }^{4,5}$ Various 
clinical rehabilitation approach was developed in order to ameliorate this symptom. ${ }^{6}$ Virtual reality (VR) application for assessment and treatment can potentially enhance current rehabilitation approaches' benefits and lead to long-lasting improvements in USN patients. ${ }^{7,8}$ However, the treatment effectiveness of using VR for clinical use required more evidence. $^{9}$

The use of head-mounted display (HMD) has been introduced for a clinical assessment of left USN and showed greater accuracy than the standard clinical test. ${ }^{10,11}$ The HMD might present diverse visual information to evaluating the objective disturbance of visual space recognition. ${ }^{12}$ Several techniques have been implemented to simulate neglect symptoms in healthy subjects, ${ }^{13}$ e.g., wedge prism adaptation on postural control behavior. ${ }^{14}$ Additionally, there was a strong association between postural disorders and spatial neglect in stroke patients., 45,16 The HMD with a tilted web camera was estimated to affect a visual perception to coordinating hand and eye movements in performing a reaching task. ${ }^{17}$ The static and dynamic balance in a virtual environment is perceived through an HMD and physical environment. ${ }^{18,19}$ Furthermore, the use of HMD with eyesopened affects an increase of the body sway during a standing posture. ${ }^{20}$

In order to explore postural behavior in the USN condition, this study develops an HMD modification as the USN model with potential application in a clinical context. The model to replicate neglect experience is challenging. Furthermore, to reduce clinical risk in developing a new approach, this model was needed to understand the physiopathological mechanism in healthy subjects before implementing the developed treatment to an actual USN patient to recover neglect symptoms, including postural balance. Regarding outcomes, we had two main hypotheses. First, we hypothesized that center of pressure $(\mathrm{CoP})$ measurements in standing balance control when the healthy subjects were using this USN model are valid and reliable at different times. Second, we hypothesized that the subjects in this mimic USN model have a postural balance profile is resembling the actual USN conditions. Therefore, the present study aimed to estimate the validity and test-retest reliability of CoP measurements in static standing balance (SSB) and dynamic standing balance (DSB) of healthy participants were using the USN model and to examine whether this model's use influenced postural balance control.

\section{METHODS}

\section{Participants}

Sixty-four healthy participants consisting of 26 females and 38 males (mean age: 27.9 years, bodyweight: $59.9 \mathrm{~kg}$, height: $166.1 \mathrm{~cm}$ ) participated in this experiment. All participants were right-handed, stated healthy physical conditions, and gave written informed consent before the test sessions. The study procedures followed are in agreement with the ethical standards of the responsible human experimentation committee (Approval number by the Tokyo Metropolitan University ethical committee, 19075) and with the 1975 Declaration of Helsinki, revised in 2000.

\section{Experimental protocol}

The HMD (VR headset for mobile phone with 3D glasses) and smartphone (Galaxy S6 edge, SCV31, Samsung Electronics Japan Co., Ltd) with a web camera (SVPro VR 3D camera) were used. To emulate a mild left USN model condition, we 
modified web camera visual direction installed on HMD of 10 degrees tilted to the right to shift visual information space more inclined to the right side only (Fig. 1). Participants who wore glasses may wear or took them off as long as they can see clearly without feeling dizzy and did not disturb HMD and its web camera use. Participants were conditioned as a non-USN model at the first test with web camera visual direction was centered as standard view. In comparison, it was modified as the left USN model condition at the second test.

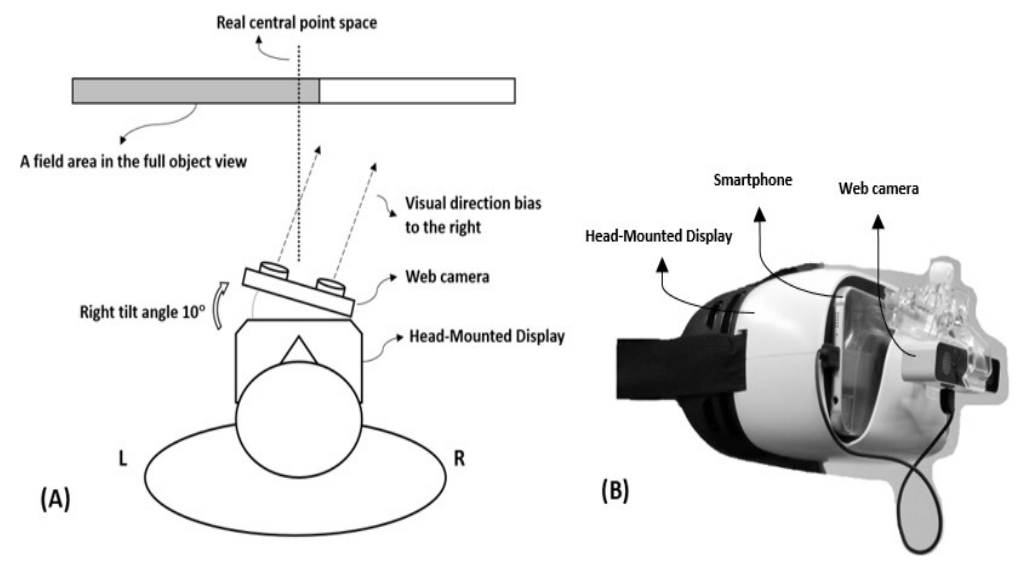

Figure 1. (A) HMD and web camera illustration as left USN model. L, left; R, right; (B) HMD and web camera device.

The experiment was administered in a quiet laboratory environmental situation. In this study, standing balance control was defined as SSB and DSB. The SSB test measured the subject's ability to stand upright with both arms folded across the chest and both feet resting on top of the CoP force plate with eyes-opened for 30 seconds. The DSB to the left $(L)$ and right $(R)$ tests measured the subject's ability to sway their posture in the frontal plane (leftward and rightward) as possible by keeping an idle position perpendicular and stable for 30 seconds each. To reducing fatigue and risk of falling and considering the procedure feasibility if the tests are applied to the actual patient, each test in a short duration of 30 seconds was performed. ${ }^{21}$ A portable CoP force plate (SR Vision by Sumitomo Riko Co. Ltd, Japan) was used to measure the CoP displacement (Fig. 2). The frequency of the signals was recorded at the sampling rate of $20 \mathrm{~Hz}$ to generate the CoP data analysis. The obtained coordinates data is high-pass filtered and displayed.

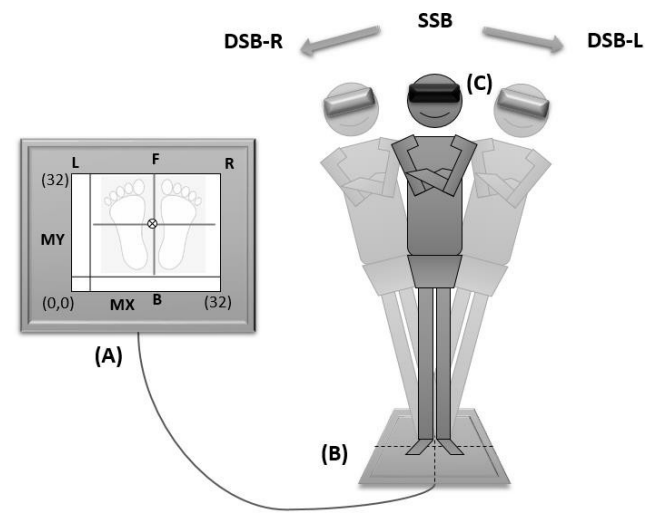

Figure 2. Apparatus illustration which used for standing balance tests. (A) CoP monitor on the computer software; (B) CoP platform device (SR Vision by Sumitomo Riko Co. Ltd, Japan); (C) HMD and web camera. $L$, left; $R$, right; $F$, front; $B$, back. 
Figure $2 \mathrm{~A}$ illustrated the CoP monitor on the computer software. The data of coordinates point position that displayed on the corner of the left side bottom indicated the lowest value of $\mathrm{CoP}$ measurement quantitatively $(0,0)$. This study quantified the CoP alteration average on the $\mathrm{X}$-axis $(\mathrm{MX})$ and $\mathrm{Y}$-axis $(\mathrm{MY})$ that indicated CoP displacement in the medial-lateral (ML) and anterior-posterior (AP) planes with a maximum value of 32 , respectively. The CoP length that indicated postural stability was calculated as a CoP trajectory in centimeters for 30 seconds. The bilateral load ratio (bodyweight) that indicated postural orientation was calculated as an average of the percentage ratio of the right and left foot force pressure on the CoP platform surface.

Two physical therapists as evaluators monitored participants in trials of the tests performed. In the first test, the SSB test was performed one-time with eyes-opened. The DSB tests to the right and the left (eyes-opened) were performed two times for each direction in order of $R-L-L-R$, sequentially. We quantified the mean results for each direction of the DSB-R and DSB-L tests. The second test was performed in the same order as in the first test. The distance between the two legs was adjusted comfortably. Specific instruction to fit the inside of the foot is to be parallel, not less than $15 \mathrm{~cm}$ precisely, for each participant to minimize CoP results variability that generates bias was performed. Participants wore the model continuously during all test sessions. No specific task was required of the participants. They were not instructed to move and visually attended to a particular field. They were allowed to sit for a moment as a resting time after the first test without changing their feet' position. The 4 modification of a web camera's direction was not informed to the participants at the second test as the USN model. The time interval between two intraday measurements of the first and second-test was less than 5 minutes approximately. The reliability tests' (first and second measurements) that indicated data variability was performed on a different day after a month on average.

\section{CoP measurement analysis}

The mean and standard deviation (mean \pm SD) of the CoP-ML, CoP-AP, CoP length, and left load ratio of the SSB and DSB to the right and left were displayed on each outcome measurement. The bodyweight ratio to the right and left side of the body was assumed to be opposite and vice versa, so that we presented only the left load ratio in tables. The positive and negative values of a changing ratio percentage between the nonUSN and USN model conditions indicated an increase and decrease in CoP measurement parameters. The CoP-ML value decreasing indicated that CoP moved to the leftward in the $\mathrm{ML}$ axis and vice versa. The CoP-AP value decreasing indicated that CoP moved backward in the AP axis and vice versa. The CoP length value reduction indicated that postural control became more stable and vice versa.

\section{Statistical Analysis}

The data were composed using Microsoft excel software after file format conversion from SR Vision platform original software. Descriptive statistics were calculated to characterize the sample. All mean $\pm S D$ of the CoP-ML, CoP-AP, CoP length were presented in centimeters $(\mathrm{cm})$. The bilateral bodyweight load ratio was presented in percentage (\%). The relationship between the unit of measure and the total number of measurements of the same variable was calculated for concurrent validity tests. The regression analysis of Pearson's correlation and Spearman's rank correlation was 
performed after the data normality test. The single measure intraclass correlation coefficient (ICC $(1,1))$ and their 95\% confidence intervals $(\mathrm{Cl})$ based on the one-way random-effects model to estimate the consistency of test-retest reliability of CoP outcome measurement were performed. ${ }^{22}$ The model of ICC used was a random model, in which the same subject was measured twice by one examiner.

The CoP data was collected as the first test (non-USN model) and second test (USN model) to evaluate the USN model use influences standing balance control by a comparison test. The paired sample t-test and Wilcoxon signed ranks test were performed to compare both test conditions after the data normality test. The statistical power of the effect size of Cohen's d between two conditions was estimated. ${ }^{23}$ The agreement between the non-USN and USN model tests was examined with Bland-Altman plots for the CoP measurement, with defined limits of agreement of $1.96 \mathrm{SD}$ above and below the mean difference. The difference in CoP measurement between the non-USN model and the USN model against mean measurement was plotted. The statistical software (IBM Corp. SPSS V. 26) was used, and the significance level was set at $p<0.05$ (significance) and $p<0.01$ (Very significant).

\section{RESULTS}

\section{Participants characteristics}

Table 1 presented the participants' characteristics of each analysis group. 16 $(25 \%)$ subjects of 64 participants were randomly selected from those who agreed to be measured twice 5 were involved two times measurement for the test-retest reliability analysis. The data collection period was administered approximately over six months for all participants. The time interval between two inter-day visits of first and second measurements was a median of 41 days.

Table 1 - Participants characteristics

\begin{tabular}{llccc}
\hline Analysis group & Gender & $\begin{array}{c}\text { Age (years) } \\
\text { Mean } \pm S D\end{array}$ & $\begin{array}{c}\text { Weight }(\mathrm{kg}) \\
\text { Mean } \pm S D\end{array}$ & $\begin{array}{c}\text { Height }(\mathrm{cm}) \\
\text { Mean } \pm S D\end{array}$ \\
\hline $\begin{array}{l}\text { USN model validity andcomparison } \\
\begin{array}{l}\text { analysis } \\
(\mathrm{N}=64)\end{array}\end{array}$ & $\begin{array}{l}\text { Male }(\mathrm{n}=38) \\
\text { Female }(\mathrm{n}=26)\end{array}$ & $27.9 \pm 4.9$ & $59.9 \pm 7.9$ & $166.1 \pm 6.3$ \\
$\begin{array}{l}\text { USN model reliability analysis } \\
(\mathrm{N}=16)\end{array}$ & $\begin{array}{l}\text { Male }(\mathrm{n}=10) \\
\text { Female }(\mathrm{n}=6)\end{array}$ & $27.4 \pm 5.0$ & $60.8 \pm 8.7$ & $166.8 \pm 6.9$ \\
\hline
\end{tabular}

Note: USN, Unilateral Spatial Neglect

\section{CoP measurement validity and test-retest reliability}

Table 2 presented the CoP measurement concurrent validity test. All CoP variables have shown valid results, but not on the CoP-ML and left load ratio in the DSB-R test. Table 3 reported the test-retest reliability $(\operatorname{ICC}(1,1))$ on a different day of CoP measurement. The results indicated that ICC estimation tended to more reliable when subjects performed the DSB test than the SSB test in the non-USN and USN model conditions. The CoP-ML in the SSB and DSB tests tended to be consistent in moderate to good reliability in both conditions. However, the CoP-AP in the SSB and DSB tests tended to be consistent in poor to moderate reliability in both conditions. The CoP length and the left load ratio showed poor reliability in the non-USN model in the SSB test. In contrast, 
Brazilian Journal of Motor Behavior

they tended to be consistent in moderate to excellent reliability in both conditions in the DSB tests.

Table 2 - Non-USN and USN model CoP measurement concurrent validity test $(\mathrm{N}=64)$

\begin{tabular}{lll}
\hline Variables & $\begin{array}{l}\text { Non-USN model } \\
\left(\mathrm{R}^{2}\right)\end{array}$ & $\begin{array}{l}\text { USN model } \\
\left(\mathrm{R}^{2}\right)\end{array}$ \\
\hline SSB & $.693^{* *}$ & $.715^{* *}$ \\
CoP-ML & $.894^{* *}$ & $.908^{* *}$ \\
CoP-AP & $.672^{* *}$ & $.612^{* *}$ \\
CoP length & $.602^{* *}$ & $.757^{* *}$ \\
Left load ratio (\%) & & \\
DSB - R & .131 & .117 \\
CoP-ML & $.926^{* *}$ & $.916^{* *}$ \\
CoP-AP & $.930^{* *}$ & $.879^{* *}$ \\
CoP length & .023 & -0.050 \\
Left load ratio (\%) & & \\
DSB - L & $.484^{* *}$ & $.492^{* *}$ \\
CoP-ML & $.934^{* *}$ & $.946^{* *}$ \\
CoP-AP & $.932^{* *}$ & $.929^{* *}$ \\
CoP length & $.512^{* *}$ & $.502^{* *}$ \\
Left load ratio (\%) & &
\end{tabular}

Table 3 - CoP test-retest reliability $(\mathrm{ICC}(1,1))$ on a different day in standing balance $(\mathrm{N}=16)$

\begin{tabular}{|c|c|c|c|c|c|c|}
\hline \multirow[b]{2}{*}{ Variables } & \multicolumn{3}{|l|}{ Non-USN model } & \multicolumn{3}{|l|}{ USN model } \\
\hline & $\begin{array}{c}1 \text { st measurement } \\
(\text { Mean } \pm S D)\end{array}$ & $\begin{array}{c}2^{\text {nd }} \text { measurement } \\
(\text { Mean } \pm S D)\end{array}$ & ICC (95\% Cl) & $\begin{array}{c}1 \text { st measurement } \\
(\text { Mean } \pm S D)\end{array}$ & $\begin{array}{c}2^{\text {nd }} \text { measurement } \\
(\text { Mean } \pm S D)\end{array}$ & ICC (95\% Cl) \\
\hline \multicolumn{7}{|c|}{ r } \\
\hline CoP-ML & $15.96 \pm 0.46$ & $16.08 \pm 0.54$ & $0.548(0.10,0.81)$ & $15.93 \pm 0.99$ & $15.65 \pm 0.74$ & $0.535(0.09,0.81)$ \\
\hline CoP-AP & $17.18 \pm 1.18$ & $17.81 \pm 1.64$ & $0.221(-0.28,0.63)$ & $16.91 \pm 1.49$ & $17.52 \pm 1.46$ & $0.439(-0.04,0.76)$ \\
\hline CoP length & $13.13 \pm 2.99$ & $14.25 \pm 4.04$ & $0.471(0.00,0.78)$ & $14.74 \pm 4.34$ & $14.73 \pm 4.93$ & $0.764(0.46,0.91)$ \\
\hline Left load ratio (\%) & $50.62 \pm 2.00$ & $49.87 \pm 1.93$ & $0.258(-0.24,0.66)$ & $50.98 \pm 4.17$ & $51.46 \pm 3.45$ & $0.722(0.38,0.89)$ \\
\hline \multicolumn{7}{|l|}{ DSB - R } \\
\hline CoP-ML & $22.01 \pm 1.21$ & $22.42 \pm 1.52$ & $0.741(0.41,0.90)$ & $22.33 \pm 1.83$ & $22.66 \pm 1.58$ & $0.852(0.63,0.95)$ \\
\hline CoP-AP & $16.94 \pm 1.32$ & $17.31 \pm 1.38$ & $0.250(-0.25,0.65)$ & $16.56 \pm 1.47$ & $17.03 \pm 1.81$ & $0.501(0.04,0.79)$ \\
\hline CoP length & $23.71 \pm 12.74$ & $23.50 \pm 8.18$ & $0.872(0.68,0.95)$ & $23.89 \pm 12.03$ & $25.32 \pm 10.95$ & $0.914(0.78,0.97)$ \\
\hline Left load ratio (\%) & $21.13 \pm 4.80$ & $18.63 \pm 5.55$ & $0.644(0.25,0.86)$ & $20.21 \pm 6.55$ & $17.44 \pm 5.93$ & $0.804(0.54,0.93)$ \\
\hline \multicolumn{7}{|l|}{ DSB - L } \\
\hline CoP-ML & $9.88 \pm 1.55$ & $9.32 \pm 2.13$ & $0.860(0.65,0.95)$ & $9.69 \pm 1.85$ & $9.08 \pm 1.97$ & $0.849(0.63,0.94)$ \\
\hline COP-AP & $16.88 \pm 1.80$ & $17.35 \pm 1.32$ & $0.328(-0.17,0.70)$ & $16.95 \pm 1.87$ & $17.70 \pm 1.81$ & $0.378(-0.11,0.73)$ \\
\hline CoP length & $24.55 \pm 13.93$ & $23.82 \pm 9.70$ & $0.728(0.39,0.89)$ & $25.17 \pm 12.22$ & $24.32 \pm 9.69$ & $0.810(0.55,0.93)$ \\
\hline Left load ratio (\%) & $80.17 \pm 7.40$ & $82.20 \pm 8.21$ & $0.868(0.67,0.95)$ & $80.79 \pm 8.15$ & $83.10 \pm 7.39$ & $0.868(0.67,0.95)$ \\
\hline
\end{tabular}

Note: USN, Unilateral Spatial Neglect; SSB, Static Standing Balance; DSB, Dynamic Standing Balance; R, Right; L, Left; CoP, Centre of Pressure; ML, Medial-Lateral; AP, Anterior-Posterior; ICC, Intraclass Correlation Coefficient; $\mathrm{Cl}$, Confidence Intervals. 


\section{Non-USN model and USN model comparison}

Table 4 presented the comparison of CoP measurement between the non-USN model and the USN model. Figure 3 illustrated the CoP displacement in the AP and ML plane of the normal condition (non-USN model) to the USN model condition. The results showed that when the subjects performed the SSB test, the CoP-AP moved backward ($1.40 \%)$ significantly with a small effect size $(p<0.05)$, while the CoP length increased $(7.67 \%)$ significantly with a small to medium effect size $(p<0.05)$. Furthermore, the CoPAP moved backward $(-1.5 \%)$ very significantly on performing the DSB-R test with a small effect size $(p<0.01)$. In comparison, it moved forward $(1.86 \%)$ very significantly on performing the DSB-L test with a small effect size $(p<0.01)$. However, the left load ratio has shown an insignificant difference in all measurements $(p>0.05)$. Figure 4 showed Bland-Altman plots for the CoP measurement agreement comparisons. No many points lie outside the limits (less than $50 \%$ ).

Table 4 - Non-USN and USN model CoP measurement comparison ( $\mathrm{N}=64$

\begin{tabular}{|c|c|c|c|c|c|}
\hline Variables & $\begin{array}{l}\text { Non-USN model } \\
\text { (Mean } \pm S D)\end{array}$ & $\begin{array}{l}\text { USN model } \\
\text { (Mean } \pm S D)\end{array}$ & $\begin{array}{l}\text { Changing ratio } \\
(\%)\end{array}$ & $p$-value & Cohen's d \\
\hline \multicolumn{6}{|l|}{ SSB } \\
\hline CoP-ML & $16.09 \pm 0.56$ & $15.96 \pm 0.89$ & -0.80 & $0.059^{*}$ & 0.172 \\
\hline CoP-AP & $17.27 \pm 1.50$ & $17.03 \pm 1.60$ & -1.40 & $0.027^{* *}$ & 0.156 \\
\hline CoP length & $14.05 \pm 3.43$ & $15.13 \pm 4.27$ & 7.67 & $0.038^{* *}$ & -0.278 \\
\hline Left load ratio (\%) & $49.83 \pm 2.34$ & $50.18 \pm 4.33$ & 0.70 & 0.187 & -0.100 \\
\hline \multicolumn{6}{|l|}{ DSB - R } \\
\hline CoP-ML & $22.27 \pm 1.41$ & $22.32 \pm 1.72$ & 0.22 & 0.566 & -0.031 \\
\hline CoP-AP & $17.12 \pm 1.53$ & $16.86 \pm 1.72$ & -1.50 & $0.006^{* * *}$ & 0.157 \\
\hline CoP length & $23.27 \pm 8.70$ & $23.11 \pm 9.17$ & -0.70 & 0.522 & 0.018 \\
\hline Left load ratio (\%) & $19.53 \pm 5.96$ & $19.18 \pm 6.88$ & -1.80 & 0.223 & 0.055 \\
\hline \multicolumn{6}{|l|}{ DSB - L } \\
\hline CoP-ML & $9.76 \pm 1.86$ & $9.64 \pm 1.95$ & -1.23 & 0.147 & 0.063 \\
\hline CoP-AP & $17.09 \pm 1.66$ & $17.41 \pm 1.84$ & 1.86 & $0.001^{* * *}$ & -0.181 \\
\hline CoP length & $23.51 \pm 10.12$ & $24.09 \pm 9.30$ & 2.50 & 0.279 & -0.060 \\
\hline Left load ratio (\%) & $80.81 \pm 7.80$ & $81.04 \pm 8.14$ & 0.29 & 0.329 & -0.029 \\
\hline
\end{tabular}

Note: USN, Unilateral Spatial Neglect; SSB, Static Standing Balance; DSB, Dynamic Standing Balance; R, Right; L, Left; CoP, C of Pressure; ML, Medial-Lateral; AP, Anterior-Posterior. Paired sample t-test or Wilcoxon signed ranks test was used for compar test; ${ }^{*} p<0.1$ (Tendency); ${ }^{* *} p<0.05$ (Significance); ${ }^{* * *} p<0.01$ (Very significant).

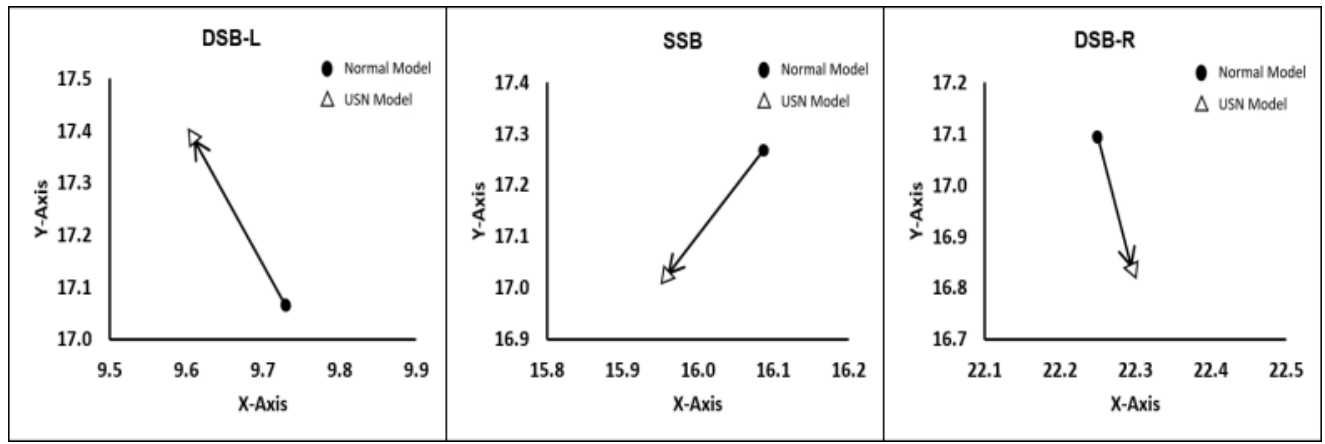

Figure 3. CoP displacement indicated by ML, Medial-Lateral (X-Axis) and AP, Anterior-Posterior (YAxis) (cm) 


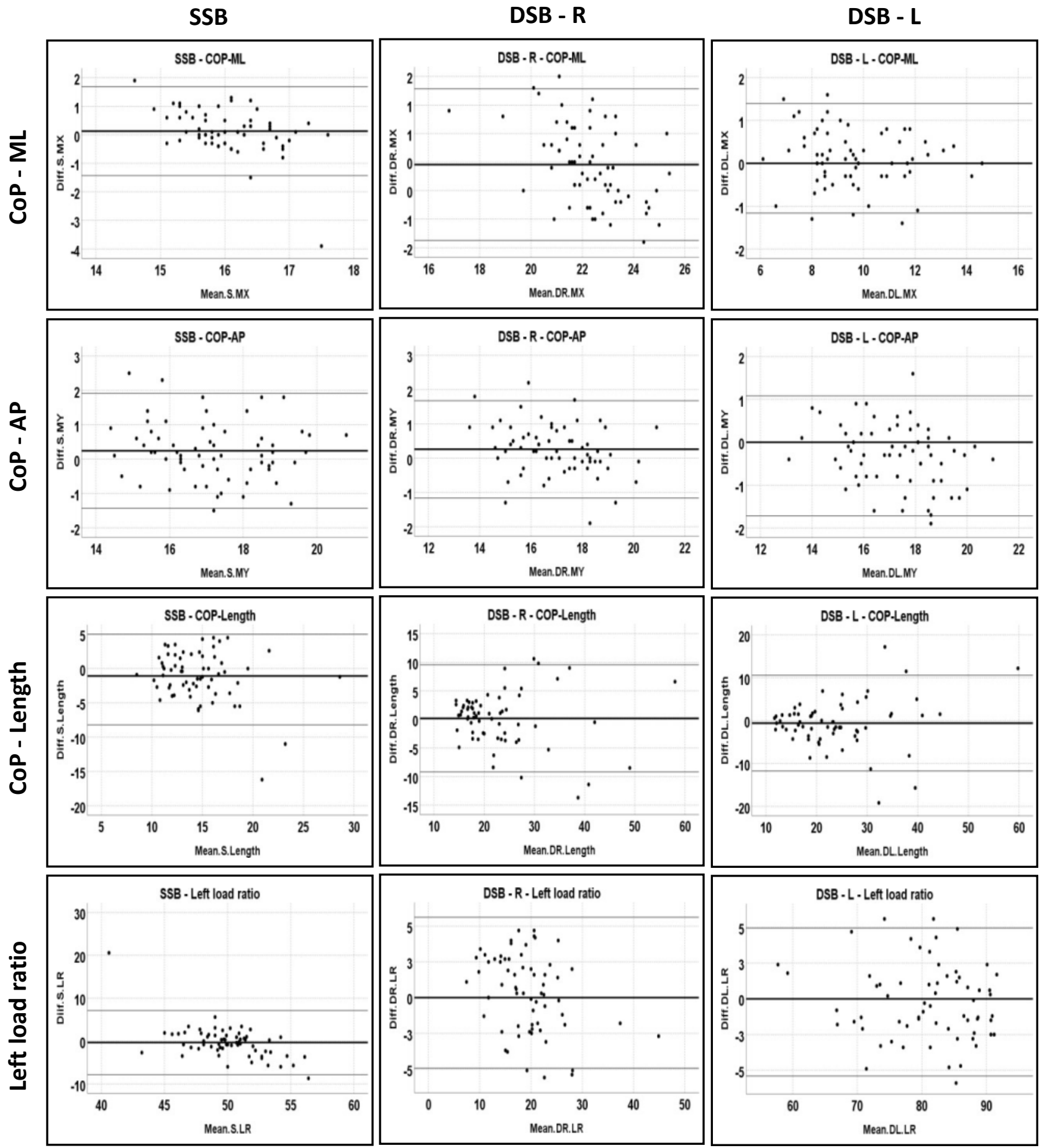

Figure 4. Bland-Altman plots are representing agreement comparisons between CoP measurements of non-USN and USN model conditions $(\mathrm{N}=64)$. The $\mathrm{Y}$-axis of the plot corresponds to the difference between the two CoP measurement systems, while the $X$-axis is the average of the two CoP measurements $(\mathrm{cm})$. USN, Unilateral spatial neglect; SSB, Static standing balance; DSB, Dynamic standing balance; R, Right; L, Left; CoP, Center of Pressure; ML, Medial-Lateral; AP, AnteriorPosterior; Diff, Difference. 


\section{DISCUSSION}

The present study was intended to develop the HMD with a modified web camera as a left USN model in healthy participants. This study was aimed to estimate the validity and reliability of center of pressure (COP) measurements in SSB and DSB of healthy participants were using the USN model and to examine whether this model's use influenced postural balance control. In general, our findings were confirmed our hypotheses. So far, no previous study elaborating on this USN model use concerning postural balance control. Moreover, as a new model, such protocols' validity and test-retest reliability have not been established. Although healthy participants have no clinical symptoms, our trial emulated the USN condition made by this model to be similar to an actual visuospatial neglect situation. In our procedure, the projected modification could shift the visual information in extrapersonal space more incline to the right side of the body in which was considered to resemble a specific sign of spatial neglect condition that has the spontaneous horizontal deviation of the eyes and head.2,4,18 Clinically, USN patients also suffered body weakness in the contralateral side extremities that affected the shifts of the CoP position, postural instability, and weight-bearing asymmetry. $5,15,24$

Regarding the outcomes, most CoP measurements have excellent concurrent validity except CoP-ML and Left load ratio on performing the DSB-R test (Table 2). This result appeared because its value increased to follow the postural sway that moved in the same direction by the test conducted and might be related to a modified web camera direction. Furthermore, the CoP reliability results showed that most of the CoP variables of non-USN model conditions indicated large variability in the SSB test (Table 3). In comparison to the previous studies elucidated that improved CoP measures were present on the second visit, indicating a potential learning effect as an external perturbation response. ${ }^{21,25}$ Moreover, the CoP-ML variability decreased while CoP-AP increased in hemiparesis conditions accompanied by restricted vision but not healthy controls. ${ }^{26}$ However, our results suggested that the USN model conditions generally produced more reliable $\mathrm{CoP}$ measures than non-USN conditions during tests.

Moreover, the CoP-AP variable in two measurement times showed poor reliability in the present study. The DSB tests of the USN model showed small variability except for the CoP-AP in the DSB-L test. This inverse tendency proved that the non-USN condition experienced more convenience and had reserve capacity to adjust and alter postural orientation in any direction. 18,19 In this case, maintaining postural balance on the SSB test is considered more straightforward than the DSB test. Hence, our results suggested the USN model conditions with interfered visual situations due to tilted web camera modifications showed a restricted response to adjust their postural balance in a difficult task of bilateral DSB tests. So that CoP variability data appeared to more reliable in the USN model but not in the sagittal plane. This condition might account for the test's characteristic in a mediolateral plane that may come from noise instead of real physiological effects.

Based on the results, the sagittal plane CoP point altered significantly compared to the frontal plane in both SSB and DSB measurements when subjects performed as the USN model (Table 4). However, the CoP-ML is more reliable than CoP-AP in two measurement times (Table 3). Compare well with Michel et al. (2003) and Nijboer et al. 
(2014), the phenomenon of the CoP moving in AP direction occurs in opposite vector between two DSB tests (Fig. 3). Moreover, an unstable posture response occurred in the SSB test when subjects perform as the model. These responses were presumed as an anticipatory postural adjustment (APAs) mechanism to adapt unusual reception of visual input perturbation experienced by participants as the USN model.5,16,20

Furthermore, our results confirmed that healthy subjects presented a small average across tests of bodyweight distribution. ${ }^{27}$ Corresponding to Ishii et al. (2010) and Mansfield et al. (2013), the bodyweight load ratio was not changed significantly in USN patients. However, our results showed that a bilateral shift fluctuation is unidirectional with CoP displacement to the right or left 28 Regarding limits of agreement (Fig. 4), the points on the Bland-Altman plots were scattered all over the place, above and below zero, then it suggested that there is no consistent bias of one measurement versus the other. Hence, it showed a good agreement between the non-USN and USN model in several CoP measurements associated with the comparison tests, such as the CoP-ML and left load ratio in the SSB test, and the CoP-ML, CoP length, and left load ratio in both DSB tests.

Practically, this USN model visual environment may not yet fully described the USN situation's actual physiopathological mechanisms. However, it resembled the actual USN appearance in visual attention and postural balance experience. Further research scheme is needed to explore the clinical application of this model. Besides, beyond our current CoP testing paradigm, while taking into account the validity and reliability findings of this study, sensorimotor adaptation also needed to consider before mimicking movement in healthy individuals. ${ }^{13,26}$ As a fundamental study, the translation of our work into clinical practice was to propose using this model in healthy participants to develop new assessment and treatment simulation feasibility in further preliminary trials, in the framework to understand physiological effects to improve neglect symptoms, including postural balance, before applied in the neuro-rehabilitation context with minimal risks.13,14

The present study has some limitations. The ergonomic impression and an unconscious response of the head and trunk rotation degrees were not examined in detail. Although it is considered sufficient as participant representation, we included only 16 (out of $64,25 \%$ ) subjects in test-retest reliability estimation. The interval between the two interday visits was more than a month apart. Moreover, all participants merely started with the non-USN model and followed the USN model to determine whether this order affected results. Trials tested only one time per condition as the first test (non-USN model) and second test (USN model). Currently, varieties of HMD devices available in the community have been used clinically. The HMD use is promising if it can digitally control the level of visual shift with a more sophisticated setup. Then, our procedure paradigm needed further trials using various more advanced equipment in the future. Hence, future study design should address these issues to concern.

\section{CONCLUSION}

In conclusion, although the CoP-ML and the left load ratio on performing the DSB$R$ test were not valid, the other CoP measurements were valid to measure standing balance control with a good agreement between the non-USN and USN models. In contrast, the CoP measurements in the ML plane were more reliable than in the AP plane to measure standing balance control in two times measurement. Furthermore, the USN 
model CoP length and left load ratio were more reliable than in the non-USN model on performing the SSB test. While performing the DSB-R and DSB-L tests, they were reliable to measure standing balance control in two times measurement. Additionally, with a good agreement among two times CoP measurement comparisons of non-USN and USN model conditions, the left USN model use appeared to modulate changes in postural adaptation and adjustment in the CoP length on performing the SSB test and in the CoP-AP on performing the SSB and DSB tests due to deviation of visual direction as the immediate effect of use. These outcomes may rely on visual sensory input and internal postural body representation as human postural balance control mechanisms. Further research might explore the USN model used in sitting position, sit to stand, and gait performance. We suggested an advanced study to notice ergonomic aspects, examine the right USN model group, and add a web camera rotation degree presumed to affect postural balance changes.

\section{REFERENCES}

1. Heilman KM, Valenstein E. Mechanisms Underlying Hemispatial Neglect. Ann Neurol. 1979;5:166-70. doi.org/10.1016/j.jijhcs.2016.04.004

2. Swan L. Unilateral Spatial Neglect. Phys Ther. 2001;81(9):1572-80.

3. Ishii F, Matsukawa N, Horiba M, Yamanaka T, Hattori M, Wada I, et al. Impaired ability to shift weight onto the non-paretic leg in right-cortical brain-damaged patients. Clin Neurol Neurosurg. 2010;112(5):406-12.

4. Nijboer TCW, Ten Brink AF, Van Der Stoep N, Visser-Meily JMA. Neglecting posture: Differences in balance impairments between peripersonal and extrapersonal neglect. Neuroreport. 2014;25(17):1381-5.

5. Gottlieb D, Levine DN. Unilateral Neglect Influences the Postural Adjustments After Stroke. J Neuro Rehab. 1992;6(1):35-41.

6. Meidian AC, Wahyuddin, Amimoto K. Rehabilitation interventions of unilateral spatial neglect based on the functional outcome measure: A systematic review and meta-analysis. Neuropsychol Rehabil. 2020;1-30.

7. Corbetta D, Imeri F, Gatti R. Rehabilitation that incorporates virtual reality is more effective than standard rehabilitation for improving walking speed, balance and mobility after stroke: A systematic review. J Physiother. 2015;61(3):117-24.

8. Tsirlin I, Dupierrix E, Chokron S, Coquillart S, Ohlmann T. Uses of Virtual Reality for Diagnosis, Rehabilitation and Study of Unilateral Spatial Neglect: Review and Analysis. CYBER Psychol Behav. 2009;12(2):175-81.

9. Ogourtsova T, Souza Silva W, Archambault PS, Lamontagne A. Virtual reality treatment and assessments for post-stroke unilateral spatial neglect: A systematic literature review. Neuropsychol Rehabil. 2017;27(3):409-54.

10. Tanaka T, Ifukube T, Sugihara S, Izumi T. A case study of new assessment and training of unilateral spatial neglect in stroke patients: effect of visual image transformation and visual 
stimulation by using a head mounted display system (HMD). J Neuroeng Rehabil. 2010;7(20):1-8.

11. Kim JH, Lee BH, Go SM, Seo SW, Heilman KM, Na DL. Improvement of hemispatial neglect by a see-through head-mounted display: a preliminary study. J Neuroeng/ Rehabil. 2015;12(114):1-6.

12. Sugihara S, Tanaka T, Miyasaka T, Izumi T, Shimizu K. Assessment of visual space recognition of patients with unilateral spatial neglect and visual field defects using a head mounted display system. J Phys Ther Sci. 2016;28(2):332-8.

13. Michel C. Simulating unilateral neglect in normals: Myth or reality? Restor Neurol Neurosci. 2006;24(4-6):419-30.

14. Michel C, Rossetti $Y$, Rode G, Tilikete $C$. After-effects of visuo-manual adaptation to prisms on body posture in normal subjects. Exp Brain Res. 2003;148(2):219-26.

15. Pérennou D. Postural disorders and spatial neglect in stroke patients : A strong association. Restor Neurol Neurosci. 2006;24(4-6):319-34.

16. Lamontagne A, Paquet N, Fung J. Postural adjustments to voluntary head motions during standing are modified following stroke. Clin Biomech. 2003;18(9):832-42.

17. Numao T, Fujita Y, Ichikawa K, Ide S, Katori H, Shimada T, et al. Leftward Optical Shift Induces Bias in Line Bisection: A Study with Healthy Subjects Using a Head-mounted Display. Prog Rehabil Med. 2019;4(20190008):1-6.

18. Lubetzky A V., Wang Z, Krasovsky T. Head mounted displays for capturing head kinematics in postural tasks. J Biomech. 2019;86:175-82.

19. Robert MT, Ballaz L, Lemay M. The effect of viewing a virtual environment through a headmounted display on balance. Gait Posture. 2016;48:261-6.

20. Imaizumi LFI, Polastri PF, Penedo T, Vieira LHP, Simieli L, Navega FRF, et al. Virtual reality head-mounted goggles increase the body sway of young adults during standing posture. Neurosci Lett. 2020;737:14-9.

21. van den Hoorn W, Hodges PW, van Dieën JH, Kerr GK. Reliability of recurrence quantification analysis of postural sway data. A comparison of two methods to determine recurrence thresholds. J Biomech. 2020;107:109793.

22. Koo TK, Li MY. A Guideline of Selecting and Reporting Intraclass Correlation Coefficients for Reliability Research. J Chiropr Med. 2016;15(2):155-63.

23. Lakens D. Calculating and reporting effect sizes to facilitate cumulative science: A practical primer for t-tests and ANOVAs. Front Psychol. 2013;4:1-12.

24. Mansfield A, Danells CJ, Zettel JL, Black SE, Mcllroy WE. Determinants and consequences for standing balance of spontaneous weight-bearing on the paretic side among individuals with chronic stroke. Gait Posture. 2013;38(3):428-32.

25. Robbins SM, Caplan RM, Aponte DI, St-Onge N. Test-retest reliability of a balance testing protocol with external perturbations in young healthy adults. Gait Posture. 2017;58(July):433-9. 
26. Kuramatsu Y, Yamamoto Y, Izumi SI. Sensorimotor strategies in individuals with poststroke hemiparesis when standing up without vision. Motor Control. 2020;24(1):15067.

27. Prado-Rico JM, Duarte M. Asymmetry of body weight distribution during quiet and relaxed standing tasks. Motor Control. 2019;23(4):553-65.

28. Marigold DS, Eng JJ. The relationship of asymmetric weight-bearing with postural sway and visual reliance in stroke. Gait Posture. 2006;23(2):249-55.

\section{ACKNOWLEDGMENTS}

The authors' thanks to all participants, laboratory staff in the Graduate School of Human Health Science, and the Tokyo Human Resources Fund for City Diplomacy Scholarship Program at Tokyo Metropolitan University for supporting this study.

Citation: Meidian AC, Yige S, Lesmana SI, Amimoto K. (2021). Validity and reliability of standing balance control measurement on the left unilateral spatial neglect model. Brazilian Journal of Motor Behavior, 15(3): 167-179. Editors: Dr Fabio Augusto Barbieri - São Paulo State University (UNESP), Bauru, SP, Brazil; Dr José Angelo Barela São Paulo State University (UNESP), Rio Claro, SP, Brazil; Dr Natalia Madalena Rinaldi - Federal University of Espírito Santo (UFES), Vitória, ES, Brazil.

Copyright:C 2021 Meidian, Yige, Lesmana and Amimoto and BJMB. This is an open-access article distributed under the terms of the Creative Commons Attribution-Non Commercial-No Derivatives 4.0 International License which permits unrestricted use, distribution, and reproduction in any medium, provided the original author and source are credited.

Funding: This work was supported by the Tokyo Human Resources Fund for City Diplomacy Scholarship Program at Tokyo Metropolitan University.

Competing interests: The authors have declared that no competing interests exist.

DOI: https://doi.org/10.20338/bjmb.v15i3.247 